\title{
Analysis of the Impact of Abrasive Jet's Concentration on the External Flow Field
}

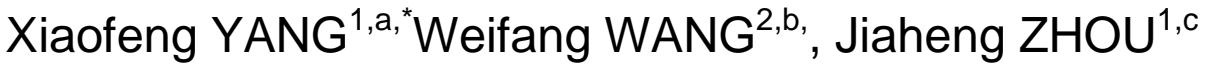

\author{
${ }^{1}$ Shenzhen Institute of Wuhan University, Shenzhen,518057, China \\ ${ }^{2}$ Shenzhen Nanxing Marine Engineering Services Co., Ltd, Shenzhen,518067, China \\ axfyang@whu.edu.cn, ${ }^{\mathrm{b}}$ wfwang@163.com, ${ }^{\mathrm{C}}$ jiaheng161@126.com \\ ${ }^{*}$ Corresponding author
}

Keywords:Abrasive jet, particles concentration, impact on the external flow field, hitting power Abstract: The technology of abrasive jet assisted drilling has the advantages of high efficiency and low energy consumption, which causesits wider application in the field of offshore oil and gas exploitation engineering.This paper used fluid analysis software to simulate and analyze the movement of abrasive jet under submerged condition, studied how the particles concentration and other factors affect the abrasive jet'shitting efficiency. Using gambitto model a two-dimensional nozzle, simulating in the numerical simulation software fluent, the study found that the change of abrasive concentration could affect the hitting efficiency of the abrasive jetsignificantly and when the abrasive particle size was 0.2 andthe abrasive concentration was $70 \%$, the hitting efficiency was the biggest.

\section{Introduction}

The application of abrasive jet is greatly superior to traditional water jetin the marine oil and gas drilling. When cutting the same material, the required water pressure of abrasive jet is lower than that of traditional water jet because of the impact of massive abrasive particles against the cutting material [1]. Marine oil and gas are frequently exploitedfrom the bottom of the sea where the water pressure is very high. Because of the unique characteristics of submerged jets, traditional water jet hardly work at the bottom of the sea, which causes a more general use of abrasive jet in the field of marine oil and gas exploitation [2-3].

This paper used fluid analysis software to simulate and analyze the movement of abrasive jet under submerged condition, studiedhow the particles concentration and other factors affect the abrasive jet'shitting efficiency.

Using gambit to model a two-dimensional nozzle, simulating in the numerical simulation software fluent, calculating and comparing the results, this paper studied the factors affecting the hitting efficiency of abrasive jet and worked out effective measures to improve it.

\section{Fluid-solid coupling analysis of abrasive jet external flow field}

Taking speed entrance in place of pressure entrance as boundary condition during the simulation,defining the abrasive density as $2600 \mathrm{~kg} / \mathrm{m}^{3}$, particle size as $0.2 \mathrm{~mm}$, the water inlet velocity as $6 \mathrm{~m} / \mathrm{s}$, abrasive inlet velocity as $1 \mathrm{~m} / \mathrm{sfor}$ the first time set, abrasive volume fraction as 0.1 , iteration timeas1000, the graph of flow field was as follows: 


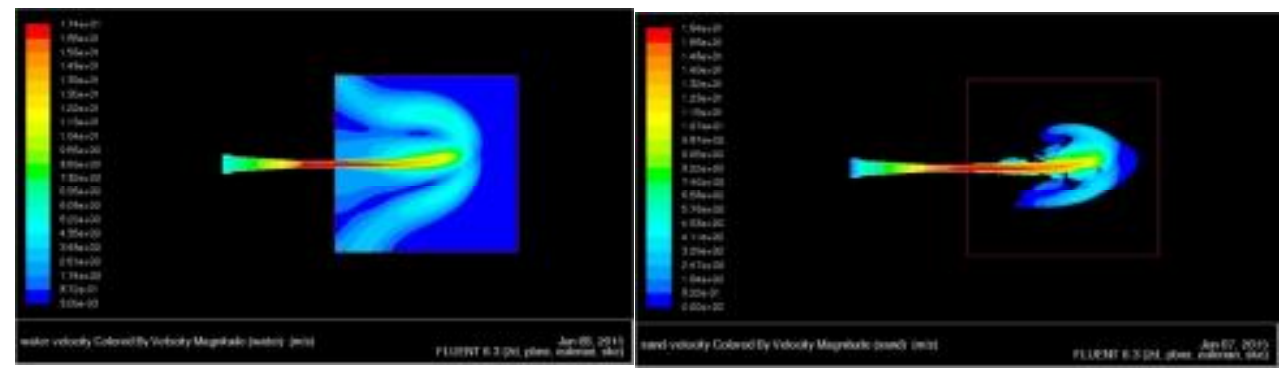

a. water flow field b.abrasive flow field

Figure1 the distribution of water flow field and abrasive

It was obvious to see that the water accelerated the abrasive as a carrier. But the maximum speed of the abrasive would never reach the speed of the water. Only the density of abrasive is bigger than that of water, the abrasive hasedges and corners and its cluster is better than water. So the appropriate mixture of water and abrasive would greatly improve the overall capacity of the abrasive jet [4-5].Despite thehighest speed of abrasivewas slightly lower than that of water, its high speed area was much closer to the external flow field, which suggested that the attenuation of abrasive's velocity was slower. Adding abrasive in the water helped to improve the jet'shitting power.

\section{The affect of abrasive concentration on the flow field}

Simulation scheme: taking speed inlet as boundary conditions during simulation,defining the abrasive density as $2600 \mathrm{~kg} / \mathrm{m}^{3}$, particle size as $0.2 \mathrm{~mm}$ and considering factors that affect the abrasive jet's hitting powerare various, the simulation defaulted other conditions such as the pump pressure, the concentration of abrasive, the abrasive particle size, the target distance, etc., and just changed the abrasive concentration to accomplish the simulation [6-7]. The abrasive volume fraction was: $0.1,0.2,0.3,0.4,0.5,0.6$, iteration time was 1000 . Water flow field distribution was as follows:

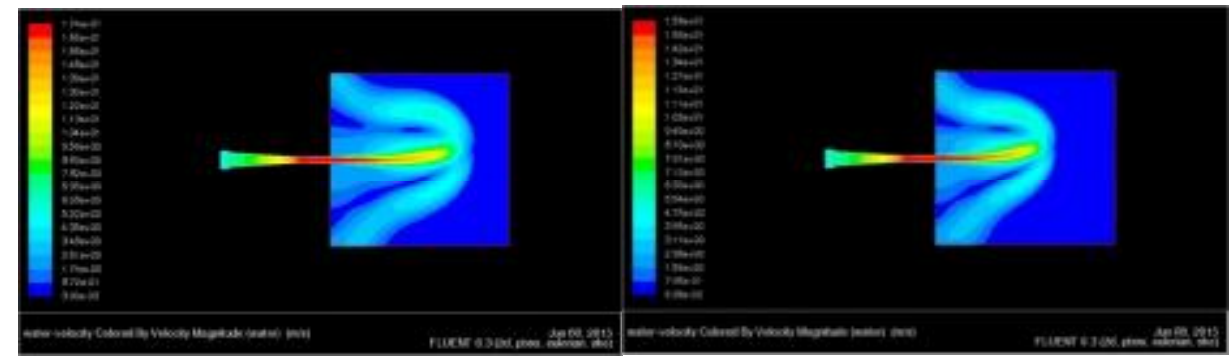

aabrasive volume fraction $0.1 \quad$ b.abrasive volume fraction 0.2

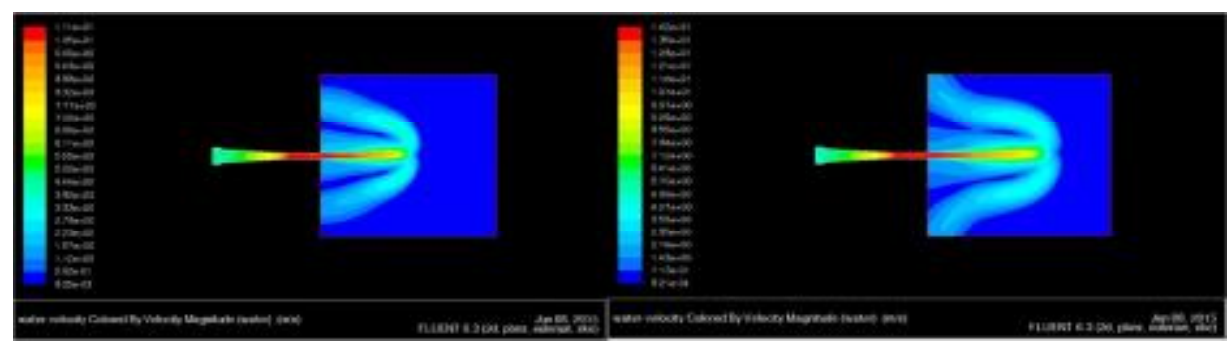

c.abrasive volume fraction 0.3 d.abrasive volume fraction 0 . 


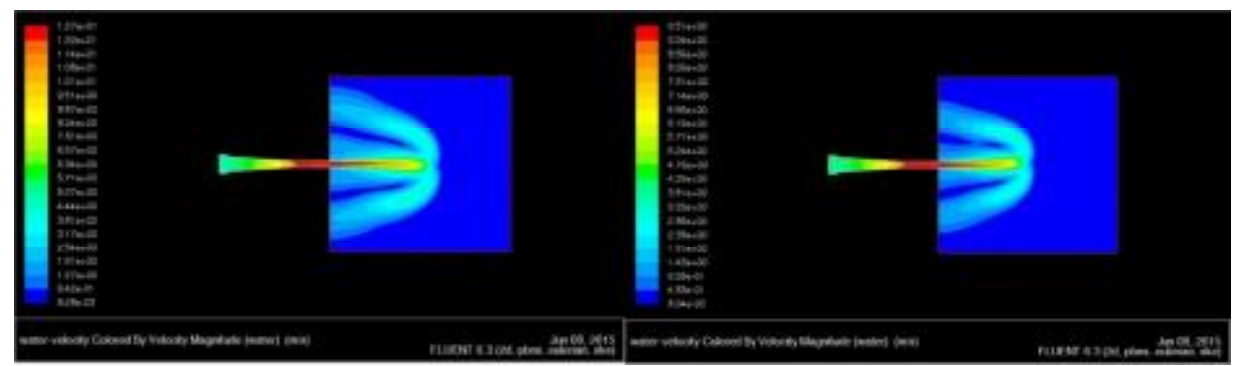

e.abrasive volume fraction 0.5

f.abrasive volume fraction 0.6

Figure 2 water flow field

Abrasive flow field distribution was as follows:

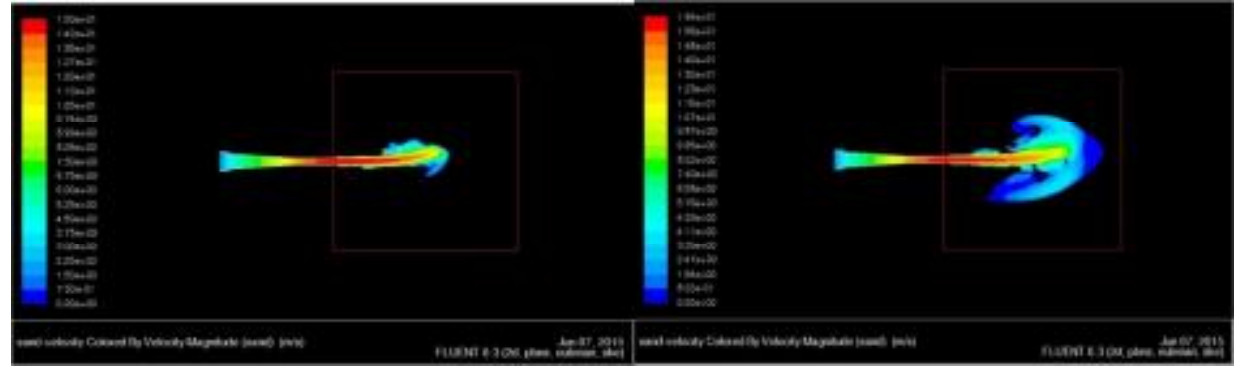

aabrasive volume fraction 0.1

b.abrasive volume fraction 0.2

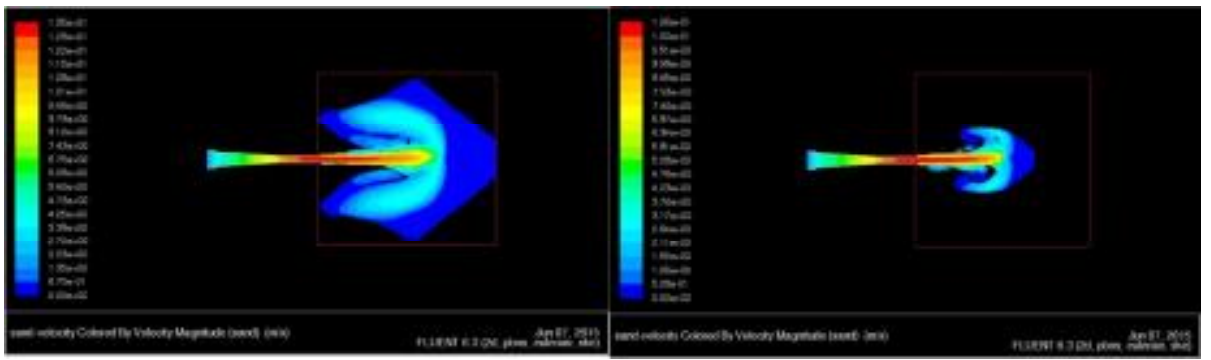

c.abrasive volume fraction 0.3 d.abrasive volume fraction 0.4

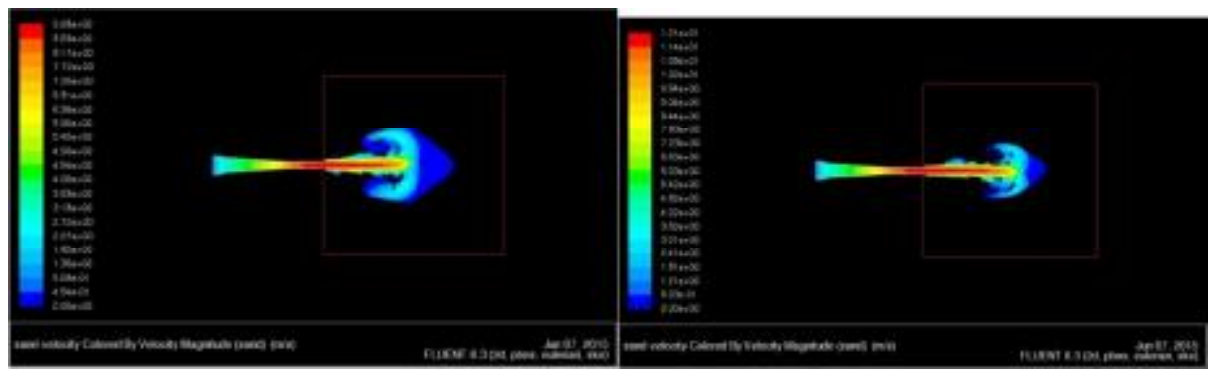

e.abrasive volume fraction 0.5 f.abrasive volume fraction 0.6

Figure 3 the distribution of abrasive

In the sight of the flow field, the maximum speed of both the water and the sand was reduced with the increasement of the volume fraction of sand, which was in line with the actual situation where the total weight of sand increased along with its volume fraction. According to the principle of conservation of energy, the maximum speed would be lower accordingly.

Besides, it can't be ignored that the increase of abrasive volume fraction would improve the frequency of abrasive particles striking targets and strengthen the jet hitting powerat the same time in practical engineering application. But the interference between the particles would reduce the hitting power of the jet when the abrasive volume fraction increased to a certain amount[8-9]. Simply using the core section of the flow speed was not enough to measure the hitting efficiency of abrasive jet, the gainingmomentum of the abrasive should also be considered. 
The calculation formula of momentum $p$ is:

$p=m v$

$p$-momentum, $m$-the weight of membrane material, $v$ - the core section speed.

When analyzing the jet with different abrasive concentrations in unit time, the ratio of the mass shot out equaled to the ratio of the concentration. For example,the mass shot out in unit time doubled when the concentration changed from $20 \%$ to $40 \%$. In order to simplify the calculation, the mass in the calculating process was replaced by a scalefactor. The core section of speed was simulated by fluent. The concentration and momentum data were as follows:

Table 1 momentum-concentration

\begin{tabular}{lllllllll}
\hline concentration & $10 \%$ & $20 \%$ & $30 \%$ & $40 \%$ & $50 \%$ & $60 \%$ & $70 \%$ & $80 \%$ \\
\hline momentum & 16.4 & 30 & 40.5 & 48.4 & 53 & 54.5 & 56 & 52 \\
\hline
\end{tabular}

The momentum varying with concentration:

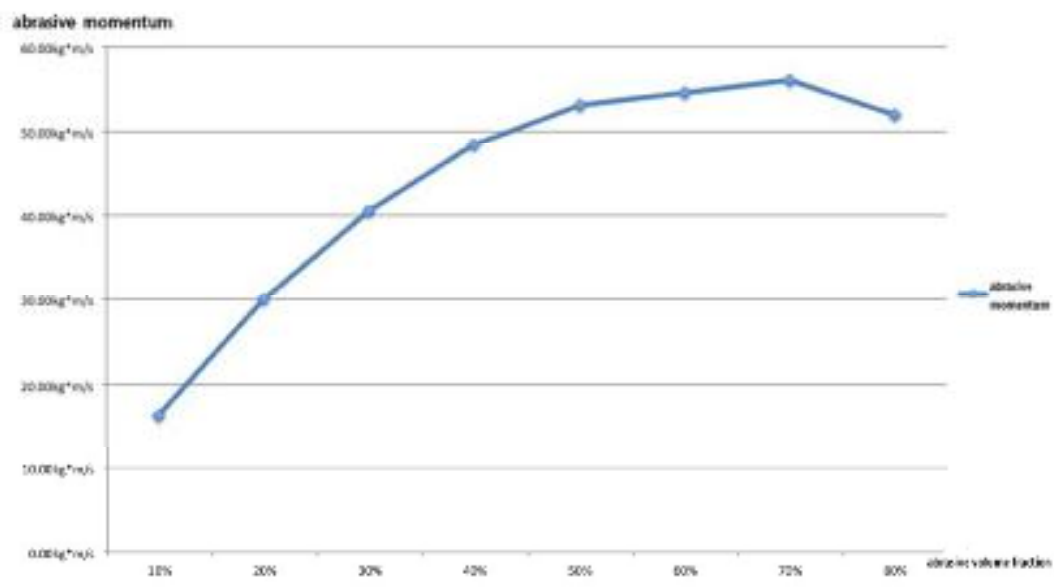

Figure 4 abrasive momentum and concentration

It was seen that the momentum of the abrasive shot out increased along with the concentration's increasementbefore the concentration reached $70 \%$. Along with the concentration approached $70 \%$, the growth of the momentum would decrease. The momentum would reach the maximum when concentration was around $70 \%$. On the other hand, when the concentration got higher than $70 \%$, the momentum began to decline. It suggested that when the concentrations washigher than $70 \%$, the abrasive started to interfere with each other because the distance between them got closer, which reduced the speed. The conclusion was that changing the concentration of the abrasive can change the hitting efficiency of the jetsignificantly. Besides, when abrasive particle size was defined as 0.2 and abrasive concentration was70\%, the hitting efficiency of the jet wasthe largest. But in engineering application, we should select abrasive concentration according to actual condition. Increasing the abrasive concentration not only improves the hitting efficiency of the jet, but also wears away the jet system, especially the nozzle.

\section{Conclusion}

We've simulated the flow field characteristics of regular abrasive jet under different abrasive concentration and granularity through fluent software. It found that the increasement of boththe concentration and thegranularity would reduce the speed of core section. But changing the concentration of the abrasive can significantly convert the momentum of the abrasive when it was 
shot out. When the concentration was less than $70 \%$, the increasement of the concentration can improve the hitting efficiency of abrasive jet. Besides, when the abrasive particle size was defined as 0.2 and abrasive concentration was $70 \%$, the hitting efficiency of the jet wasthe largest. But in engineering application, we should select abrasive concentration according to actual condition. Increasing the abrasive concentration not only improves the hitting efficiency of the jet, but also wears away the jet system, especially the nozzle.

\section{Acknowledgements}

This work is supported by the Science and Technology Projects of Shenzhen (Grant No. JSKF20150928142452831)

\section{References}

[1] Shen Zhonghou, LI Gengsheng, Wang Zhiming. New Jet theory and prospects of application in petroleum engineering. Proceedings go the 13th World Petroleum Congress, Buenos Aires, Argentina, 1991, 397 405.

[2]Yuanlin Ning, Shougen Hu. Advances in abrasive water jet, J.Lubricatingandsealing, 2002. $(01) 79 \sim 83$.

[3]Zhonghou Shen. Water jet theory and technology. University of petroleum press, Beijing, 1998.

[4]Gensheng Li, Zhongwei Huang, Shouzeng Tian. Hydraulic jet fracturing theory and application. SciencePress,Beijing, 2011.

[5]Labus T J. Fluid Jet Technology Fundamentals \& Applications. USA: American Water jet Technology Association, 1995.

[6] Wei Zhou. The Numerical Simulation of Rock Breaking Characteristics under Self-excited Oscillation Pulsed Jet. Chongqing University,Chongqing, 2014.

[7]Zhunong Liu. Numerical simulation and experimental research ofhigh pressure water jet grinding solid materials. Xi'an University of Technology, Shanxi, 2010.

[8]Kwing-So Choi, Jean-Robert DeBisschop, Brian R. Clayton. Turbulent Boundary-Layer Control by Mean of Spanwise-Wall Oscillation, J. AIAA Journal, 1998,36(7)1157-1170.

[9]Liao Zhen-fang, et al. Theory and Experimental Study of the Self-excited Oscillation Pulsed Jet Nozzle, J. Chinese Journal Mechanical Engineering, 2003, 16(4)379-383. 